\title{
Fragility of information cascades: an experimental study using elicited beliefs
}

\author{
Anthony Ziegelmeyer • Frédéric Koessler • \\ Juergen Bracht • Eyal Winter
}

Received: 23 December 2008 / Accepted: 3 December 2009 / Published online: 18 December 2009

(C) The Author(s) 2009. This article is published with open access at Springerlink.com

\begin{abstract}
This paper examines the occurrence and fragility of information cascades in two laboratory experiments. One group of low informed participants sequentially guess which of two states has been randomly chosen. In a matched pairs design, another group of high informed participants make similar guesses after having observed the guesses of the low informed participants. In the second experiment, participants' beliefs about the chosen state are elicited. In equilibrium, low informed players who observe an established pattern of identical guesses herd without regard to their pri-
\end{abstract}

This paper is a revised version of Chapter V of Ziegelmeyer's dissertation (Ziegelmeyer 2001) which resulted from a collaboration with Frédéric Koessler. It circulated earlier under the title "Behaviors and Beliefs in Information Cascades".

Electronic supplementary material The online version of this article (http://dx.doi.org/10.1007/s10683-009-9232-x) contains supplementary material, which is available to authorized users.

A. Ziegelmeyer $(\bowtie)$

Strategic Interaction Group, Max Planck Institute of Economics, Jena, Germany

e-mail: ziegelmeyer@econ.mpg.de

A. Ziegelmeyer

Faculty of Economics and Management, Technical University of Berlin, Berlin, Germany

F. Koessler

Paris School of Economics and CNRS, Paris, France

e-mail: koessler@pse.ens.fr

J. Bracht

University of Aberdeen Business School, University of Aberdeen, Aberdeen, Scotland, UK

e-mail: juergen.bracht@abdn.ac.uk

E. Winter

Department of Economics and Center for the Study of Rationality, Hebrew University of Jerusalem, Jerusalem, Israel

e-mail: mseyal@mscc.huji.ac.il 
vate information whereas high informed players always guess according to their private information. Equilibrium behavior implies that information cascades emerge in the group of low informed participants, the belief based solely on cascade guesses is stationary, and information cascades are systematically broken by high informed participants endowed with private information contradicting the cascade guesses. Experimental results show that the behavior of low informed participants is qualitatively in line with the equilibrium prediction. Information cascades often emerge in our experiments. The tendency of low informed participants to engage in cascade behavior increases with the number of identical guesses. Our main finding is that information cascades are not fragile. The behavior of high informed participants differs markedly from the equilibrium prediction. Only one-third of laboratory cascades are broken by high informed participants endowed with private information contradicting the cascade guesses. The relative frequency of cascade breaks is $15 \%$ for the situations where five or more identical guesses are observed. Participants' elicited beliefs are strongly consistent with their own behavior and show that, unlike in equilibrium, the more cascade guesses participants observe the more they believe in the state favored by those guesses.

Keywords Information cascades · Fragility $\cdot$ Elicited beliefs $\cdot$ Depth-of-reasoning analysis $\cdot$ Experimental economics

JEL Classification C72 · C92 · D82

\section{Introduction}

In recent years a great deal of attention has been focused on situations in which the existence of informational externalities leads to a loss of social welfare. In these situations, Bayesian rational individuals with limited information share that information with others through their choices. The attempt to take advantage of the information of their predecessors prevents individuals from exploiting their private information in a socially optimal way. This likely consequence of social learning is what has been called information cascades (Banerjee 1992 and Bikhchandani et al. 1992). An information cascade occurs when the accumulated evidence from previous choices is so conclusive that individuals rationally herd without regard to their private information. In an information cascade, choices do not convey private information, the benefit of diversity of information is lost, social learning stops completely and the failure of information aggregation is spectacular. Information cascades have been proposed as explanations of a variety of phenomena, such as fads, fashions, booms and crashes. ${ }^{1}$

\footnotetext{
${ }^{1}$ Since choices are the words for the transmission of information between individuals, information cascades occur only if the information space is large relative to the choice space. Smith and Sorensen (2000) provides the most comprehensive and exhaustive analysis of social learning in situations where players observe the full sequence of past choices, and establishes that the failure of information aggregation is not a robust property. However, as rightly argued by Chamley (2004), which reviews a large spectrum of economic models, the inefficiency of social learning is a robust conclusion. Acemoglu et al. (2008) addresses how the social network structure, which determines the observations of each individual, affects information aggregation.
} 
As emphasized by Bikhchandani et al. (1992) (henceforth BHW), in the Bayesian Nash equilibrium of information cascade models, the convergence of behavior is idiosyncratic and fragile. Individuals quite rapidly converge upon one choice on the basis of some but very little information. In fact, a choice is fixed upon when the accumulated evidence from previous choices grows to be just enough to overcome an individual's private information pointing in the opposite direction. The next individual is also just barely willing to ignore her private information and all further individuals do the same thing, i.e., they are in a cascade. Since choices are uninformative once a cascade has started, only the information of a few early choices is aggregated and the informativeness of a cascade does not rise with the number of similar choices. Thus, a small bulk of evidence causes the vast majority of individuals to make one choice over the other, which might be the wrong one. But the fallibility of information cascades causes them to be fragile meaning that incorrect decisions can be rapidly reversed, e.g., by the arrival of a little extra information. These two consequences of social learning are two sides of the same coin and constitute the departure point of our experimental study.

We examine the occurrence and fragility of information cascades in two laboratory experiments. In each decision making round, a random choice is made between two (almost) equally likely states but the state is not revealed to the participants until the end of the round. Low informed participants sequentially guess which state has been chosen, with each participant observing all previous guesses and a low-accuracy signal (correct with probability 2/3). In a matched pairs design, high informed participants observe the guesses of the low informed participants and a high-accuracy signal (correct with probability 4/5). Each participant is incentivized to make a correct guess. In the second experiment, participants' beliefs about the chosen state are elicited. In equilibrium, low informed players who observe an established pattern of identical guesses herd without regard to their private information whereas high informed players always guess according to their private information. Equilibrium behavior therefore implies that information cascades emerge in the group of low informed participants, the belief based solely on cascade guesses is stationary, and information cascades are systematically broken by high informed participants endowed with private information contradicting the cascade guesses.

The behavior of low informed participants is qualitatively in line with the equilibrium prediction. In situations where the equilibrium predicts a guess inconsistent with the one relying only on the private signal, low informed participants herd without regard to their private signal about $70 \%$ of the time. Consequently, information cascades often emerge in our experiments. Moreover, participants' tendency to engage in cascade behavior increases with the number of identical guesses, ranging from less than $65 \%$ after two identical guesses to $100 \%$ after seven identical guesses. On the contrary, the equilibrium poorly predicts the behavior of high informed participants. Only one-third of laboratory cascades are broken by high informed participants endowed with private information contradicting the cascade guesses. The relative frequency of cascade breaks is $15 \%$ for the situations where five or more identical guesses are observed. Our experiments show that information cascades occur in the laboratory and that as the number of identical guesses increases the fragility of laboratory cascades rapidly vanishes. This evidence is supported by our analysis of the large 
data set on elicited beliefs. Participants' elicited beliefs are strongly consistent with their own behavior and show that, unlike in equilibrium, the more cascade guesses participants observe the more they believe in the state favored by those guesses. High informed participants believe more strongly than low informed participants that the informativeness of a laboratory cascade rises with the number of identical guesses.

We attempt to understand participants' behavior by estimating an error-rate model that uses logistic response functions to determine guess probabilities and allows for different error rates on different levels of reasoning about others' behavior. The results of our depth-of-reasoning analysis suggest that participants apply medium chains of reasoning. They learn from observing their predecessors' guesses and realize that other participants also learn from observing their respective predecessors. However, participants' reasoning gets rather imprecise on the highest level since participants think that others attribute to their respective predecessors twice the participants' error rate. Additionally, low informed participants attribute a significantly higher error rate to their predecessors whereas high informed participants attribute a significantly lower error rate to their predecessors as compared with their own. Participants' behavior can be explained along the lines of our estimated model. Compared to Bayesian rational individuals, low informed participants discount the evidence conveyed by guesses which are not part of an information cascade and they do not fully discount the evidence conveyed by guesses which are part of an information cascade. The reasoning process of low informed participants explains why they do not systematically engage in cascade behavior after having observed a few identical guesses and why they do so after having observed many identical guesses. Compared to low informed participants, high informed participants do not discount the evidence conveyed by any guess, whether it is part of an information cascade or not, which explains why long herds have such a low empirical probability of collapse.

Below, we selectively survey the experimental literature on information cascades and establish that the behavioral patterns observed in our study are well in line with the existing evidence. ${ }^{2}$ The most prominent theoretical rationalizations of the experimental regularities are presented in the discussion section of this paper.

\section{Related literature}

In a seminal study, Anderson and Holt (1997) (henceforth AH) presents three experiments to investigate the emergence of information cascades in the laboratory. In the baseline experiment, there are six participants and two urns which are equally likely to be chosen. Urn $A$ contains 2 balls labeled $a$ and 1 ball labeled $b$ while urn $B$ contains 2 balls labeled $b$ and 1 ball labeled $a$. In each decision making round, one urn is randomly chosen and participants guess sequentially which urn has been chosen, with each participant observing all previous guesses and a single draw from the chosen urn (signal correct with probability $2 / 3$ ). At the end of the round, the randomly

\footnotetext{
${ }^{2}$ We focus on laboratory studies which, similarly to our study, are based on BHW's specific model. Other experimental studies relied on alternative frameworks either to distinguish between information cascades and herding (Celen and Kariv 2004) or to investigate herding in financial markets (Drehmann et al. 2005; Cipriani and Guarino 2005).
} 
chosen urn is publicly revealed and participants who guessed correctly get $\$ 2$ while those who did not get nothing. The procedures of the second experiment extend those of the baseline experiment by adding either one or two public draws after the fourth guess. The procedures of the third experiment are identical to those of the baseline experiment except that urn $A$ contains $6 a$ balls and $1 b$ ball while urn $B$ contains 5 $a$ balls and $2 b$ balls.

Though AH find clear evidence that information cascades occur in the laboratory, participants do not always rationally ignore their private signal and follow an established pattern of previous guesses. For example, in the baseline experiment, participants rationally follow the herd in only $70 \%$ of the cases. ${ }^{3}$ These deviations from equilibrium behavior are more prevalent after the observation of two identical guesses (64\% of cascade behavior) than after the observation of five identical guesses ( $80 \%$ of cascade behavior). ${ }^{4}$ From now on, we refer to the participants' reluctance to engage in cascade behavior after a few identical guesses as to the "overweight-phenomenon". Compared to the equilibrium prediction, participants seem to overweight their private information relative to the public information of their predecessors' guesses. Like in AH's baseline experiment, we observe the overweight-phenomenon in our group of low informed participants and the phenomenon basically disappears once the evidence conveyed by predecessors' guesses is strong i.e. enough participants made the same guess.

AH's second experiment helps to find out whether laboratory cascades collapse due to the public release of information. Laboratory cascades collapse only in 5 out of the 13 situations in which participants are endowed with multiple draws contradicting the established pattern of identical guesses. This evidence is also well in line with our own findings. ${ }^{5}$

In the third experiment, where signal $b$ is much more informative than signal $a$, more deviations from equilibrium behavior are observed.

Kübler and Weizsäcker (2004) examines the robustness of laboratory cascades to the introduction of costly signals in AH's baseline experiment, i.e. participants decide whether to obtain private information or not, at a small but non-zero cost. In equilibrium, only the first participant in the decision making sequence should buy a private signal and all subsequent participants should simply imitate the first guess. These predictions perform poorly in organizing the experimental data. Indeed, participants buy too many signals suggesting that they do not consider the first participant's guess strong enough evidence (this finding is clearly related to the overweightphenomenon). However, most participants follow the majority of guesses once the

\footnotetext{
${ }^{3}$ Several laboratory studies show that AH's baseline experiment results replicate. Anderson (2001) as well as Hung and Plott (2001) establish that AH's observation that information cascades occur in the laboratory is robust to changes in payoffs and experimental settings. Additional evidence in favor of the cascade phenomenon is reported by Alevy et al. (2007) which compares the behavior of market professionals from the floor of the Chicago Board of Trade with that of college students in the gain and loss domain of earnings.

${ }^{4}$ We report our own computations of the frequencies of cascade behavior since AH does not discuss the correlation of length and strength in laboratory cascades.

${ }^{5}$ Note that AH does not report on the empirical probability of collapse of laboratory cascades since their design produces very few situations of potential collapse.
} 
evidence conveyed by the predecessors' guesses is strong. Again, we observe the same phenomenon among our low informed participants.

Kübler and Weizsäcker successfully explain these observations by conducting a statistical depth-of-reasoning analysis. The estimated error-rate model does not impose the rational expectations assumption according to which players have a correct perception of other players' error rates, or that they have a correct perception of other players' perceptions of third players, and so on. Their estimation results clearly indicate that participants apply only short chains of reasoning and that the perceived error rates get larger and larger on higher levels of reasoning, pointing at a consistent underestimation of others' degree of rationality. ${ }^{6}$ Our own statistical error-rate analysis is directly borrowed from this earlier work and the comparison of the two sets of estimation results indicates that our low informed participants exhibit less distortion in the perception of their predecessors (they apply medium chains of reasoning). However, this discrepancy between the two estimated models has few noticeable consequences in terms of behavior since our low informed participants think that others attribute to their respective predecessors twice the participants' error rate.

Goeree et al. (2007) extends AH's baseline experiment by considering long sequences of guesses, respectively 20 and 40, and two values of the signal accuracy, respectively $5 / 9$ and $6 / 9$. Their experimental results can be summarized as follows: (i) Pure laboratory cascades, i.e. two identical guesses not canceled out by previous guesses which induce herd behavior for the remaining participants in the sequence, are rarely observed. The occurrence of pure laboratory cascades decreases with the length of the sequence of guesses and increases with the signal's accuracy; (ii) Laboratory cascades are almost always broken by participants with contradictory private signals (signals pointing in the opposite direction to the accumulated evidence from previous guesses); (iii) The longer a pure laboratory cascade the more likely participants with contradictory private signals herd; and (iv) In the majority of cases, after an incorrect laboratory cascade is broken the new laboratory cascade which emerges is a correct one, i.e. laboratory cascades are self-correcting.

Goeree et al.'s results and our own results complement each other. First, the third result corroborates and extends to long sequences of guesses our finding that participants engage more in cascade behavior after longer herds. ${ }^{7}$ Second, our finding that the fragility of laboratory cascades rapidly vanishes as the number of identical guesses increases indicates that Goeree et al.'s third result is valid not only when participants are endowed with signals they should disregard but is also valid when participants are endowed with signals they should not disregard. Of course, the fact that long herds have a low empirical probability of collapse does not imply that laboratory cascades cannot collapse at all. Still, laboratory cascades are not fragile in the theoretical sense which might be a concern for social welfare (though Goeree et al. rarely observe pure laboratory cascades, they report an empirical probability of collapse of less than $10 \%$ after a dozen of identical guesses in case the signal accuracy is 6/9). In the discussion section of this paper, we come back to this issue.

\footnotetext{
${ }^{6}$ Kraemer et al. (2006) evaluates private information acquisition behavior in an experimental setting with two signal accuracies and obtains similar results.

${ }^{7}$ Kübler and Weizsäcker (2005) also documents this regularity and shows that the correlation of length and strength in pure laboratory cascades is robust to changes in experimental cascade games.
} 
The remainder of the paper is structured as follows. Section 2 outlines the theoretical model and predictions. Section 3 discusses the experimental design. In Sect. 4 we give an exhaustive analysis of participants' guesses and belief reports. Section 5 presents some theoretical rationalizations of the behavioral patterns observed in information cascade experiments. Section 6 concludes.

\section{An information cascade game with low and high informed players}

In this section we present a simple information cascades game based on BHW's specific model. First we consider a low informed setup in which the unique equilibrium outcome is characterized by a high occurrence of information cascades. Next we introduce a high informed player to show that information cascades are theoretically fragile.

\subsection{Low informed setup}

There are two payoff-relevant states of Nature (henceforth states) — state $\alpha$ and state $\beta$, two signals - signal $a$ and signal $b$, and two possible guesses_- "guess state $\alpha$ " denoted by $A$ and "guess state $\beta$ " denoted by $B$. Players have a common prior belief on the state space $\{\alpha, \beta\}$ where $\operatorname{Pr}(\alpha)=1-\operatorname{Pr}(\beta)=0.55$. $^{8}$

Nature moves first and chooses a state which remains unknown to the players. We denote this state by $\omega$ and refer to it as the true state. Players have to guess whether $\omega=\alpha$ or $\omega=\beta$. Players make their guesses in sequence and the order in which players make their guesses is exogenously specified. Player 1 guesses in period 1 , player 2 guesses in period 2, and so forth. Before making his guess, each player $i$ both observes a single draw from an urn, which constitutes his private signal $t_{i} \in\{a, b\}$, and the public history of guesses of all preceding players $1,2, \ldots, i-1$. If the state is $\alpha$ then each player draws a ball from an urn which contains two $a$ balls and one $b$ ball. If the state is $\beta$ then each player draws a ball from an urn which contains two $b$ balls and one $a$ ball. Therefore, conditionally on the true state, players' signals are i.i.d. and the conditional probabilities are given by $\operatorname{Pr}\left(t_{i}=a \mid \omega=\alpha\right)=\operatorname{Pr}\left(t_{i}=b \mid\right.$ $\omega=\beta)=2 / 3$ and $\operatorname{Pr}\left(t_{i}=a \mid \omega=\beta\right)=\operatorname{Pr}\left(t_{i}=b \mid \omega=\alpha\right)=1 / 3$. Guessing the true state, i.e., making guess $A$ in state $\alpha$ or making guess $B$ in state $\beta$, yields 10 whereas guessing the wrong state yields -5 .

For $i \geq 2$, let $\{A, B\}^{i-1}$ be the space of all possible period $i$ histories of guesses chosen by the $i-1$ predecessors of player $i$. Denote by $h^{i-1}$ an element of $\{A, B\}^{i-1}$, i.e., $h^{i-1}$ is a sequence of guesses up to player $i-1$. Let $\mu_{i}:\{a, b\} \times\{A, B\}^{i-1} \rightarrow$ $[0,1]$ be player $i$ 's belief (conditional probability given past observed guesses and his private signal) that the state is $\alpha$. Player $i$ 's belief is given by

$$
\mu_{i}\left(t_{i}, h^{i-1}\right)=\frac{\operatorname{Pr}\left(t_{i} \mid \alpha\right) \operatorname{Pr}\left(\alpha \mid h^{i-1}\right)}{\operatorname{Pr}\left(t_{i} \mid h^{i-1}\right)},
$$

\footnotetext{
${ }^{8}$ Contrary to BHW's specific model, the two payoff-relevant states are not equally likely. This difference has the advantage that tie-breaking rules are unnecessary which implies that the equilibrium outcome is unique (for more details, see Koessler and Ziegelmeyer 2000).
} 
Table 1 Probability of no cascade after any even number of players lower than or equal to eight
Number of players

Probability of no cascade

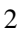

6

8
0.222

0.049

0.011

0.002

where probabilities are computed with respect to players' strategies and the prior. Given history $h^{i-1}$, signal $t_{i}$ and belief $\mu_{i}\left(t_{i}, h^{i-1}\right)$, player $i$ 's expected utility is given by $15 \mu_{i}\left(t_{i}, h^{i-1}\right)-5$ (respectively $\left.15\left(1-\mu_{i}\left(t_{i}, h^{i-1}\right)\right)-5\right)$ if his guess is $A$ (respectively $B$ ).

In equilibrium, players update their beliefs in a Bayesian rational way by observing their signal and their predecessors' guesses and they maximize their expected utility given these beliefs. Player 1 guesses in accordance with his signal. If player 2 observes an $A$ guess then he guesses $A$ as well even if his private signal is $b$. As the same argument applies for all the rest of the sequence, it is here that an information cascade results. On the contrary, if player 2 observes a $B$ guess then he predicts in accordance with his signal. If player 3 observes two $B$ guesses then he follows his predecessors' guesses even if he is endowed with an $a$ signal. This implies that the rest of the sequence joins the herd. Once a cascade has started, further guesses are uninformative. In other words, after an $A$ guess not canceled out by previous guesses, whatever their positions in the sequence, the beliefs of two followers are identical if endowed with the same private signal. Similarly, after two $B$ guesses not canceled out by previous guesses, whatever their positions in the sequence, the beliefs of two followers are identical if endowed with the same private signal.

The only history which does not lead to an information cascade is $B A B A B A \ldots$. Table 1 reports the probability of having no information cascade after any even number of players lower than or equal to eight. There is a less than $5 \%$ probability that the fifth player's guess in the sequence depends on his signal.

\subsection{High informed setup}

In this setup we assume that one and only one of the players receives a more informative signal about the state. We use the subscript $j \neq i$ to refer to this player and we denote his signal $t_{j} \in\left\{a_{S}, b_{S}\right\}$. If the state is $\alpha$ then player $j$ draws a ball from an urn which contains four $a$ balls and one $b$ ball. If the state is $\beta$ then player $j$ draws a ball from an urn which contains four $b$ balls and one $a$ ball. Therefore, player $j$ 's signal is more accurate than player $i$ 's signal: $\operatorname{Pr}\left(t_{j}=a_{S} \mid \omega=\alpha\right)=\operatorname{Pr}\left(t_{j}=b_{S} \mid \omega=\beta\right)=$ $4 / 5$ and $\operatorname{Pr}\left(t_{j}=a_{S} \mid \omega=\beta\right)=\operatorname{Pr}\left(t_{j}=b_{S} \mid \omega=\alpha\right)=1 / 5$.

In equilibrium, whatever his position in the sequence, player $j$ guesses in accordance with his signal. Indeed, player $j$ 's high-accuracy signal is twice as informative as player $i$ 's signal. If player $j$ observes an $A$ cascade and receives a $b_{S}$ signal then his signal overweighs the $a$ signal and the prior $\left(\mu_{j}\left(b_{S}, A\right)=0.38=\mu_{i}(b, \emptyset)\right)$, and player $j$ guesses in accordance with his signal. If player $j$ observes a $B$ cascade and receives an $a_{S}$ signal then his signal and the two $b$ signals just cancel out. This leaves 
player $j$ with a belief which equals the prior $\left(\mu_{j}\left(a_{S}, B B\right)=\operatorname{Pr}(\alpha)\right)$. Hence, the rational guess is $A$. Of course, if player $j$ either observes a $B A B A \ldots$ sequence or if player $j$ 's signal is in accordance with what he has observed then he has to follow his signal as well. The interesting result is that, whatever the type of information cascade that has started, the high informed player $j$ breaks the cascade when endowed with a contradictory signal.

\section{Experimental procedures}

Two experiments were run on a computer network at the laboratory of experimental economics in Strasbourg (LEES) using 96 undergraduate students from the University of Strasbourg. No participant had any training in game theory or economics of information. Each experiment was made up of three sessions that took between $1 \frac{1}{2}$ and $2 \frac{1}{4}$ hours. Sixteen participants took part in each session (plus one participant who acted as an assistant). Participants were randomly assigned to a computer terminal, which was physically isolated from other terminals. Communication, other than through the decisions made, was not allowed. Participants were instructed about the rules of the game and the use of the computer program through written instructions, which were read aloud by the assistant. A short questionnaire and one dry run followed. ${ }^{9}$ Participants, on average, earned approximately 126 French francs (including a show-up fee of 15 French francs), which was paid to them in cash at the end of the session. ${ }^{10}$

In the first experiment participants played the information cascades game described in Sect. 2, fifteen times in the same group. In each decision making round, we implemented this setup in the following way. We built two "lines" of participants, a "low line" and a "high line". The low line was constituted by nine participants whereas the high line was only made of seven participants. Participants in the high line were only in positions from three to nine. During a session a participant always belonged either to the low line or to the high line. At the beginning of the round, a random choice was made between state $\alpha$ and state $\beta$. The probability of choosing state $\alpha$ was $55 \%$. Participants were then chosen in a random order to observe a single draw from a selected urn. Balls tagged $a$ or $b$ were put in urns labeled $A$ and $B$ and drawn on the computer screen to represent participants' signals. In the low line, the signal's strength (which indicates the probability that the signal is correct) was equal to $2 / 3$. On each computer screen of the low line appeared a ball drawn from an urn containing 3 balls, two "correct" balls and an "incorrect" one. In the

\footnotetext{
${ }^{9}$ The dry run was added in order to give some experience to the participants about the computer program. Participants did not make guesses in this dry run. In each session, at least 22 participants read the instructions on their own, listened to the assistant reading the instructions aloud, and answered a questionnaire. The questionnaire mainly checked participants' understanding of the calculation of earnings. Participants who made mistakes in answering the questionnaire were paid 30 French francs and were replaced by participants who were randomly selected among those who made no mistakes on the questionnaire. Thus, in each session, subjects who were retained for participation in the remainder of the experiment had made no mistakes on the questionnaire.

${ }^{10} \$ 1$ was approximately 7.5 French francs at the time of the experiment.
} 
Table 2 Typical decision making round

\begin{tabular}{llll}
\hline $\begin{array}{l}\text { Period } \\
\text { in the low line }\end{array}$ & $\begin{array}{l}\text { Participant's position } \\
\text { in the high line }\end{array}$ & $\begin{array}{l}\text { Observed } \\
\text { history }\end{array}$ \\
\hline 1 & 1 & $\emptyset$ \\
2 & 2 & 1 & $\begin{array}{l}\text { 1st guess in the low line } \\
3\end{array}$ \\
3 & 3 & 2 & $\begin{array}{l}\text { 1st to 2nd guess in the low line } \\
\text { 1st to 3rd guess in the low line }\end{array}$ \\
$\vdots$ & 4 & $\vdots$ & $\vdots$ \\
9 & 9 & 7 & 1 st to 8th guess in the low line \\
\hline
\end{tabular}

high line, the signal's strength was equal to 4/5. On each computer screen of the high line appeared a ball drawn from an urn containing 5 balls, four "correct" balls and an "incorrect" one. This information structure was public knowledge as being part of the instructions which were read aloud by the assistant. Finally, participants were asked to make a guess about the identity of the selected state. Only the guesses of the participants in the low line were made public meaning that, whatever his line, each participant observed the previous guesses of the participants in the low line. So, it was public knowledge that each observed guess relied on a private information quality of $2 / 3$. Each participant received 10 French francs for a correct guess and -5 French francs otherwise. At the end of each round uncertainty about the true state was resolved to allow for controlled learning. Table 2 summarizes the progress of a typical decision making round.

Our innovative design has nice features. First, it allows us to collect a lot of data concerning the potential situations where a cascade should be broken (107 cascade breaks should have been theoretically observed). Second, one can investigate whether participants' behavior, both in the low and high lines, rely on the position in the decision queue. Third, as low and high informed participants observe the same history, a highly controlled comparison between low informed and high informed participants' behavior can be made.

In the second experiment we replicated Experiment 1 but we also elicited participants' beliefs about the randomly chosen state. For the sake of comparison of participants' behavior between the two experiments, the same random events, i.e., urns used and private signals, were maintained to run one session in each experiment.

\section{Eliciting beliefs}

In each period of the second experiment participants in the low line were asked to key in a probability vector which represents their beliefs that state $\alpha$ or state $\beta$ was randomly chosen at the beginning of the round. Participants in the high line reported their beliefs until the period of their guess. For example, if in a given round a participant in the high line held position 5 then he only reported 5 beliefs. The first elicitation of a participant's belief was made just after he received his signal and before making guess $A$ or $B$. Otherwise, participants' beliefs were elicited at the beginning of each period, i.e., after having observed the previous periods' guesses. Of course, in 
period 1, except for the participant who received a signal, participants' beliefs should reflect the prior. This procedure of beliefs' elicitation allowed us to collect a $9 \times 9$ matrix of beliefs for the low line and 7 vectors of beliefs for the high line, whose length goes from 3 to 9 , for each decision making round.

Participants' belief reports were rewarded on the basis of a quadratic scoring rule function. ${ }^{11}$ Thus, participant $i$ reported his beliefs in period $n$ by keying in a vector $\mu_{i n}=\left(\mu_{i n}^{\alpha}, \mu_{i n}^{\beta}\right)$ indicating his belief about the probability that the state randomly chosen at the beginning of the round is $\alpha$ or $\beta .{ }^{12}$ In period $n$, the payoff to participant $i$ when state $\alpha$ was randomly chosen and $\mu_{i n}$ is the reported belief vector is given by

$$
\Pi_{\alpha}=0.25-\frac{1}{8}\left(\left(1-\mu_{i n}^{\alpha}\right)^{2}+\left(\mu_{i n}^{\beta}\right)^{2}\right)=0.25\left(1-\left(\mu_{i n}^{\beta}\right)^{2}\right) .
$$

The payoff to participant $i$ when state $\beta$ was randomly chosen is, analogously,

$$
\Pi_{\beta}=0.25-\frac{1}{8}\left(\left(1-\mu_{i n}^{\beta}\right)^{2}+\left(\mu_{i n}^{\alpha}\right)^{2}\right)=0.25\left(1-\left(\mu_{i n}^{\alpha}\right)^{2}\right) .
$$

It can easily be demonstrated that this reward function provides an incentive for riskneutral participants to reveal their true beliefs about the randomly chosen state (see Murphy and Winkler 1970 for more details). ${ }^{13}$ The payoffs from the assessment task were all received at the end of the experiment. ${ }^{14}$ We made sure that the amount of money that could potentially be earned in the assessment part of the experiment was not large in comparison to the game being played. In this respect, the maximum amount that could be earned in the assessment task of Experiment 2 was only 33.75 French francs as compared to the theoretical expected payoff of the decision task: 90 French francs for a low informed participant and 100 French francs for a high informed participant. Table 3 summarizes the features of both experiments.

\section{Results}

First, we analyze participants' guesses and show that, in accordance with the equilibrium prediction, information cascades consistently develop in the low line. However,

\footnotetext{
${ }^{11}$ Belief elicitation using a quadratic scoring rule is widely employed in experimental economics (see for example Nyarko and Schotter 2002). Offerman et al. (2009) show how proper scoring rules can be generalized to modern theories of risk and ambiguity, and can become valid under risk aversion and other deviations from expected value. They also report experimental results suggesting that it is desirable to correct participants' reported probabilities elicited with scoring rules if only a single large decision is paid, but that this correction is unnecessary with many repeated decisions and repeated small payments.

${ }^{12}$ In the experiment $\mu_{i n}^{\alpha}$ and $\mu_{i n}^{\beta}$ were keyed in as numbers in $[0,100]$, so they are divided by 100 to get probabilities.

${ }^{13}$ While payoffs are maximized by a truthful revelation of beliefs, reporting equal probabilities for each state would guarantee the largest minimal payment ("secure" stated beliefs). Risk aversion could induce participants to behave in such a way. We did neither observe a bias toward flat beliefs' vectors (risk-averse participants) nor toward extreme beliefs' vectors (risk-loving participants) in our data.

${ }^{14}$ For the sake of understanding, instead of presenting (1) and (2) to the participants, we included in the instructions a table summarizing the respective payoff depending on the pair of beliefs reported and the chosen state.
} 
Table 3 Experimental design

\begin{tabular}{lllllll}
\hline & $\begin{array}{l}\text { Elicitation } \\
\text { of } \\
\text { beliefs }\end{array}$ & Line & $\begin{array}{l}\text { Total number } \\
\text { of } \\
\text { participants }\end{array}$ & $\begin{array}{l}\text { Number } \\
\text { of rounds } \\
\text { per session }\end{array}$ & $\begin{array}{l}\text { Total number } \\
\text { of } \\
\text { guesses }\end{array}$ & $\begin{array}{l}\text { Total number } \\
\text { of belief } \\
\text { reports }\end{array}$ \\
\hline Experiment 1 & No & Low & 27 & 15 & 405 & 0 \\
(3 sessions) & & High & 21 & 15 & 315 & 0 \\
Experiment 2 & Yes & Low & 27 & 15 & 405 & 3645 \\
(3 sessions) & & High & 21 & 15 & 315 & 1890 \\
\hline
\end{tabular}

these information cascades rarely collapse in the high line which contradicts equilibrium behavior. Second, we study the dynamics of elicited beliefs. Participants' elicited beliefs are strongly consistent with their own behavior and show that, unlike in equilibrium, the more cascade guesses participants observe the more they believe in the state favored by those guesses. Finally, we attempt to understand participants' behavior by conducting a depth-of-reasoning analysis.

Whereas equilibrium decisions for high informed players are unique whatever the history of guesses, there is no unique prediction off-the-equilibrium-path for low informed players. Hence, we only consider decisions following a history that could be part of an equilibrium outcome (histories are still included after out-of-equilibrium guesses as long as those guesses do not lead to a history that cannot be part of an equilibrium outcome). Similar results are obtained by considering all participants' guesses where data analyses rely on the assumption that each out-of-equilibrium guess which leads to a history that cannot be part of an equilibrium outcome is interpreted as a guess in accordance with the private signal.

A cascade situation is a situation where a guess $(A$ or $B)$ constitutes an established pattern, and a participant's signal does not coincide with the established pattern. Let $n_{i-1}$ be the number of $a$ signals less the number of $b$ signals that can be inferred from an equilibrium history $h^{i-1}$. Formally, player $i$ in the low line (respectively player $j$ in the high line) is in a cascade situation if either $n_{i-1}=1$ and $t_{i}=b$ (respectively $t_{j}=b_{S}$ ) or $n_{i-1}=-2$ and $t_{i}=a$ (respectively $t_{j}=a_{S}$ ). Given a cascade situation in period $i$, cascade behavior is observed in the low line if player $i$ guesses $A$ when $n_{i-1}=1$ and guesses $B$ when $n_{i-1}=-2$. Similarly, given a cascade situation in period $j$, cascade break is observed in the high line if player $j$ guesses $B$ when $n_{i-1}=1$ and guesses $A$ when $n_{i-1}=-2$.

We make four remarks concerning participants' guesses and beliefs before discussing in details the emergence and fragility of information cascades. First, only slightly more than half of the observed histories are equilibrium histories ( 54 percent). This evidence shows that play off-the-equilibrium-path occurs frequently, an observation made repeatedly in the experimental literature on information cascades. ${ }^{15} \mathrm{Sec}-$ ond, in all cases but three, a deviation from the equilibrium path by a low informed

\footnotetext{
${ }^{15}$ In the corresponding treatment by Anderson and Holt (1997), all six participants played according to the equilibrium in $60 \%$ of the rounds. Obviously, the longer the sequence of guesses the lower the percentage of rounds which display equilibrium behavior of all participants. Goeree et al. (2007) reports that in sequences of 40 guesses equilibrium histories are basically absent.
} 
Table 4 Relative frequencies of cascade behavior in equilibrium histories

\begin{tabular}{lll}
\hline Experiment & Session & Cascade behavior \\
\hline 1 & 1 & $85 \%$ \\
& 3 & $83 \%$ \\
& 5 & $70 \%$ \\
& Average & $79 \%$ \\
2 & 2 & $61 \%$ \\
& 4 & $44 \%$ \\
& 6 & $73 \%$ \\
& Average & $59 \%$ \\
\hline
\end{tabular}

participant reveals that participant's private signal (51 cases). This evidence shows that play off-the-equilibrium-path is informative. Third, the overall relative frequency of guesses which are neither in line with equilibrium behavior nor in agreement with the private signal is extremely small in the low line (less than 4 percent) but quite large in the high line (about 20 percent) where the two benchmarks always make similar predictions. Finally, one might argue that in the second experiment risk-averse participants have an incentive to hedge with their stated beliefs against adverse outcomes of their guesses. We find no clear evidence for hedging since elicited beliefs are systematically in line with guesses (see footnote 21) and participants' guesses in both experiments are similar. ${ }^{16}$

\subsection{Emergence of information cascades: guesses in the low line}

We denote by $n_{C S}$ the total number of cascade situations. The relative frequency of cascade behavior is the ratio $\frac{n_{C B}}{n_{C S}}$, where $n_{C B}$ is the total number of cases in which a low informed participant, placed in a cascade situation, guesses in contradiction with his signal. The relative frequencies of cascade behavior for each session in both experiments are given in Table 4. Information cascades emerge since cascade behavior is observed in $69 \%$ of the cascade situations. According to a robust rank-order test (Siegel and Castellan 1988, p. 137) on session-average relative frequencies of cascade behavior, we cannot reject the null hypothesis of no difference between the relative frequency of cascade behavior in Experiment 1 and the relative frequency of cascade behavior in Experiment 2 (two-sided test; $U$ statistic of 2.348; p-value > 0.10). ${ }^{17} \mathrm{We}$ conclude that cascade behavior in the absence of belief elicitation is not statistically different from cascade behavior in the presence of belief elicitation. In the rest of the section, we pool the data of both experiments when discussing participants' guesses in the low line.

We now examine participants' tendency to engage in cascade behavior depending on the number of identical guesses i.e. the depth of the cascade. Figure 1 represents

\footnotetext{
${ }^{16}$ The experimental evidence provided by Blanco et al. (2008) strongly suggests that hedging bias is not a serious concern in belief elicitation experiments.

${ }^{17}$ We have checked the robustness of the conclusion by conducting a two-sample t-test with equal variances and a two-sample Mann-Whitney test. Both two-sided tests on fully independent session-level data give a p-value strictly greater than 0.10 .
} 


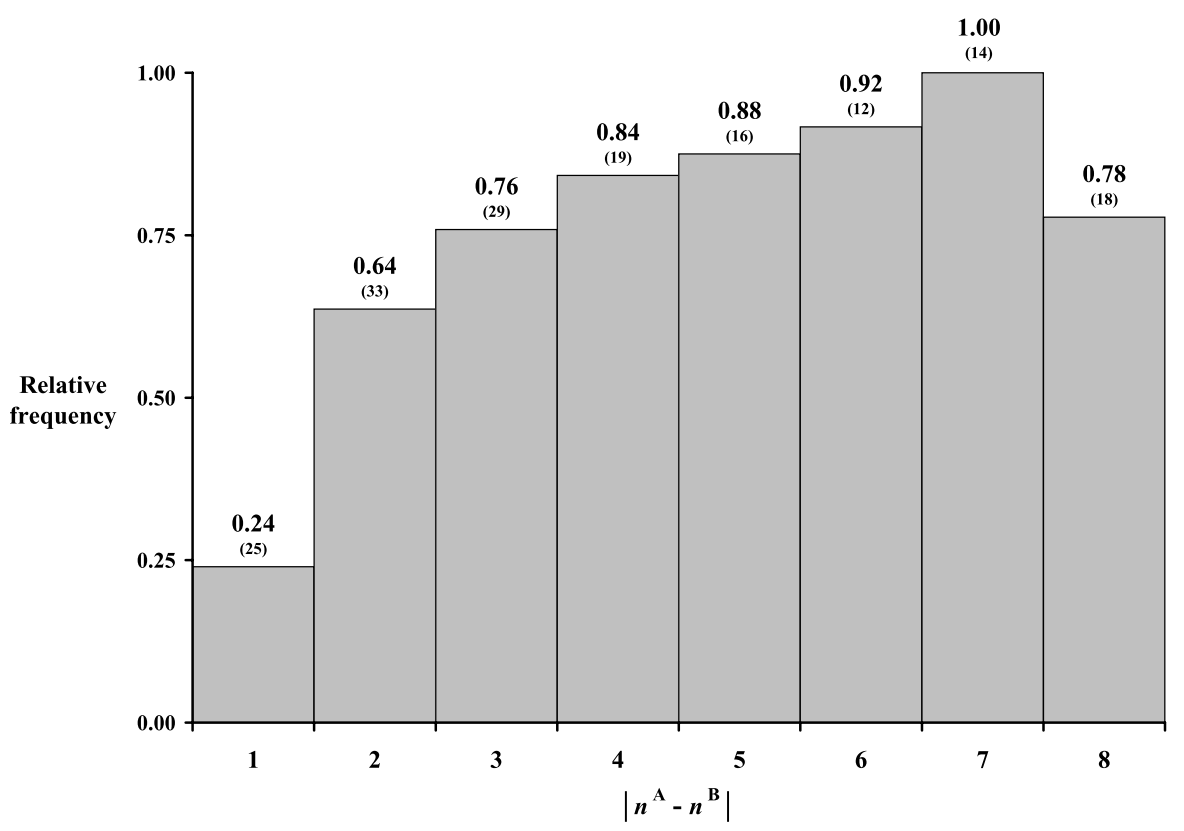

Fig. 1 Relative frequencies of cascade behavior in equilibrium histories (numbers of cascade situations are indicated in parentheses)

the relative frequencies of cascade behavior as a function of $\left|n^{A}-n^{B}\right|$, where $n^{d}$ is the number of guesses $d \in\{A, B\}$ taken up to the current period. After an $A$ guess, only $24 \%$ of the participants follow the trend when they receive a contradictory signal. After two similar guesses not canceled out by previous guesses, the relative frequency of cascade behavior increases markedly and reaches $64 \%$. When the absolute difference between the number of $A$ and $B$ guesses attains 7, the proportion of cascade behavior is identical to the theoretical one: $100 \%$ of the participants follow the established pattern. ${ }^{18}$

\subsection{Fragility of information cascades: guesses in the high line}

We define the relative frequency of cascade break as the ratio $\frac{n_{S C}}{n_{C S}}$, where $n_{S C}$ is the total number of cases where a high informed participant, placed in a cascade situation, guesses in accordance with his signal and in contradiction with the majority of previous guesses. The relative frequencies of cascade break for each session in both experiments are given in Table 5. Overall, in only one third of the situations, a high informed participant who has a signal contradicting the established pattern of guesses breaks the cascade. According to a robust rank-order test on session-average relative frequencies of cascade break, we cannot reject the null hypothesis of no difference between the relative frequency of cascade break in Experiment 1 and the relative

\footnotetext{
${ }^{18}$ In the last period, the relative frequency of cascade behavior decreases. Though we have no convincing explanation for such an anomaly, participants' elicited beliefs also reflect this "end-game" behavior (see the next subsection).
} 
Table 5 Relative frequencies of cascade break in equilibrium histories

\begin{tabular}{lll}
\hline Experiment & Session & Cascade break \\
\hline 1 & 1 & $27 \%$ \\
& 3 & $26 \%$ \\
& 5 & $53 \%$ \\
& Average & $35 \%$ \\
& 2 & $20 \%$ \\
& 4 & $42 \%$ \\
& 6 & $32 \%$ \\
& Average & $31 \%$ \\
\hline
\end{tabular}

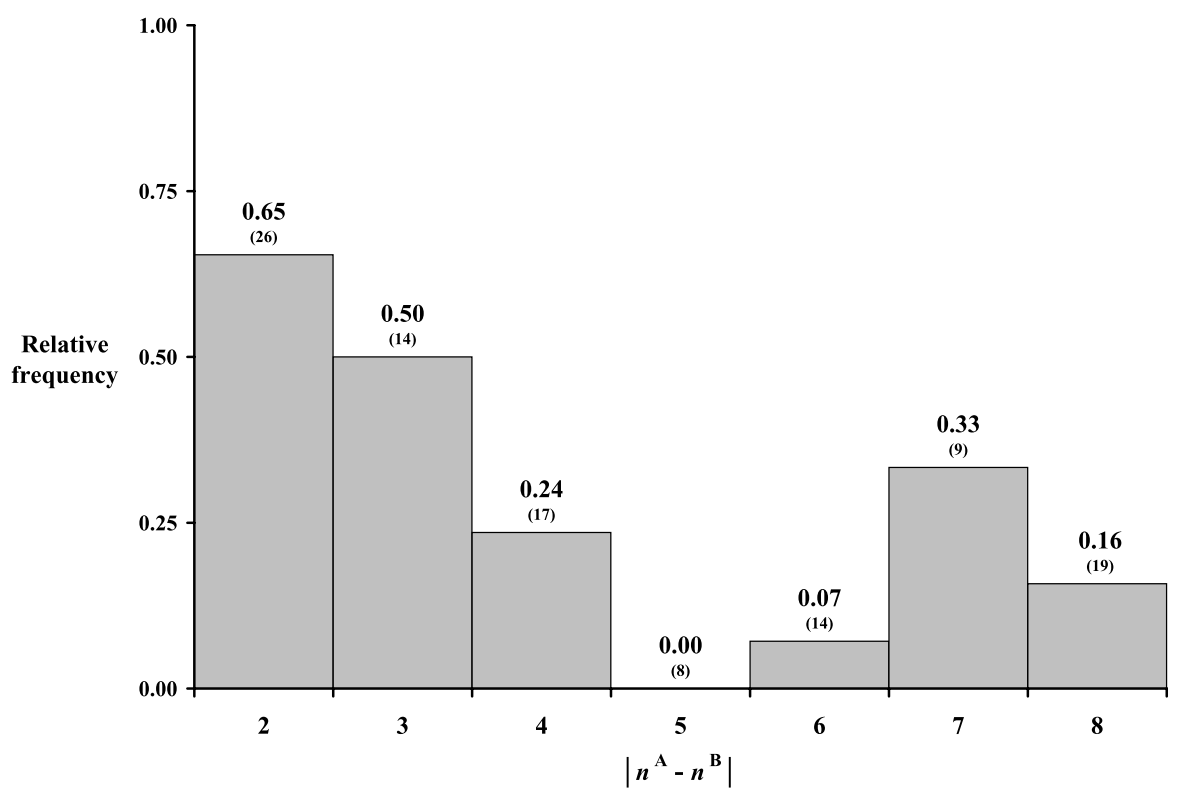

Fig. 2 Relative frequencies of cascade break in equilibrium histories (numbers of cascade situations are indicated in parentheses)

frequency of cascade break in Experiment 2 (two-sided test; U statistic of 0.182; p-value > 0.10). ${ }^{19}$ We conclude that cascade break in the absence of belief elicitation is not statistically different from cascade break in the presence of belief elicitation. In the rest of the section, we pool the data of both experiments when discussing participants' guesses in the high line.

Figure 2 shows the relative frequencies of cascade breaks depending on the depth of the cascade. At least half of the information cascades are broken in case an im-

\footnotetext{
${ }^{19}$ We have checked the robustness of the conclusion by conducting a two-sample t-test with equal variances and a two-sample Mann-Whitney test. Both two-sided tests on fully independent session-level data give a p-value strictly greater than 0.10 .
} 
Table 6 Average beliefs in the first period

\begin{tabular}{llll}
\hline & $\begin{array}{l}\text { No signal } \\
675 \text { obs. (both lines) }\end{array}$ & $\begin{array}{l}\text { Signal } a \\
26 \text { obs. (low line) }\end{array}$ & $\begin{array}{l}\text { Signal } b \\
19 \text { obs. (low line) }\end{array}$ \\
\hline $\begin{array}{l}\text { Session 2 } \\
\text { Session } 4\end{array}$ & $54 \%(0.12)$ & $78 \%(0.17)$ & $33 \%(0.22)$ \\
Session 6 & $51 \%(0.12)$ & $62 \%(0.18)$ & $36 \%(0.10)$ \\
Average elicited beliefs & $55 \%(0.14)$ & $61 \%(0.15)$ & $37 \%(0.22)$ \\
Theoretical beliefs & $53 \%(0.13)$ & $66 \%(0.18)$ & $35 \%(0.18)$ \\
\hline
\end{tabular}

balance of at most 3 guesses in one direction is observed. However, the relative frequency of cascade breaks drops to $15 \%$ when averaged over the situations where five or more identical guesses are observed. We conclude that the fragility of laboratory cascades rapidly vanishes as the number of identical guesses increases.

\subsection{Dynamics of beliefs}

In this subsection we look at the dynamics of elicited beliefs. We show that, in contradiction with equilibrium behavior but in accordance with actual behavior, participants consider cascade guesses informative.

\section{Elicited beliefs in the first period}

Table 6 summarizes the average beliefs in the first period (standard deviations are given in brackets). Those participants did not observe any guess yet but some of the low informed participants were endowed with a private signal. Actual beliefs in the first period, with or without private information, are very close to the theoretical ones. Participants seem to apply Bayes' rule when reasoning about others' guesses is unnecessary.

\section{History-dependent elicited beliefs}

Figure 3 shows the dynamics of participants' beliefs, before being endowed with a private signal, in a cascade with an established pattern of $A$ guesses (respectively $B$ guesses) when the depth of the cascade $n^{A}-n^{B}$ (respectively $n^{B}-n^{A}$ ) increases. In equilibrium, public information stops accumulating once an information cascade has started. Consequently, players' beliefs stay constant whatever the depth of the cascade (grey line). Clearly, the dynamics of stated beliefs for participants without private information do not reflect this theoretical feature (empty diamonds). Participants' beliefs increase when the depth of the cascade increases. The dynamics of actual beliefs are similar to the dynamics of "Private Information" (PI) beliefs which correspond to the beliefs players would have if it was mutually known that all players follow their private signal (full circles). 


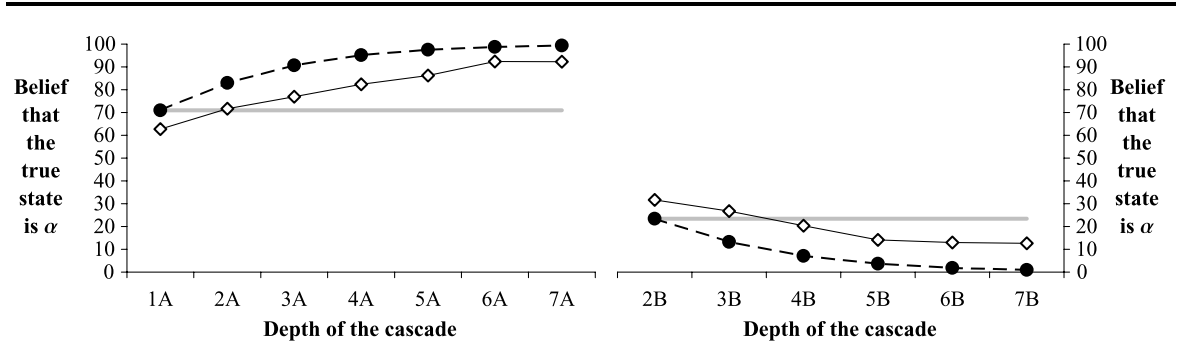

Fig. 3 Dynamics of beliefs in $A$ and $B$ cascades without private signal (low and high line)
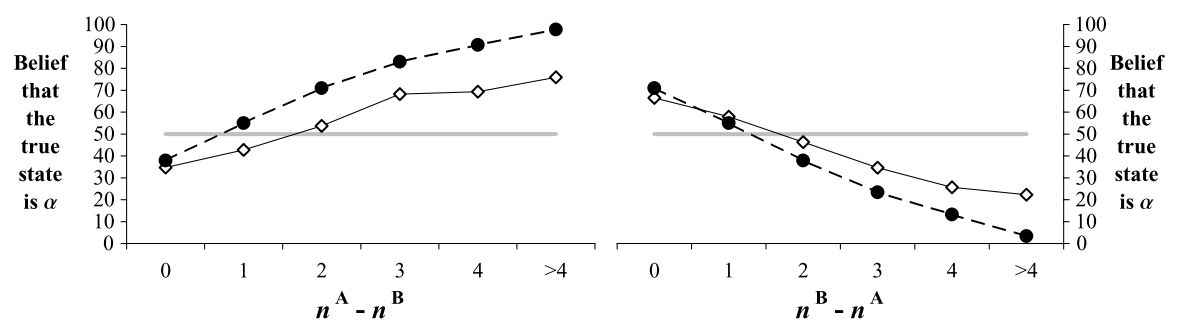

Fig. 4 Dynamics of beliefs with a weak contradictory signal (low line)

The observation that the relative frequency of cascade behavior increases with the depth of the cascade seems to be explained by the fact that participants' beliefs do not stay constant in a cascade. To see the link between the relative frequency of cascade behavior and the way participants update their beliefs we represent in Fig. 4 the dynamics of the low informed participants' beliefs when the depth of the cascade increases and their (low quality) signal contradicts the established pattern of guesses. ${ }^{20}$ The dynamics of the low informed participants' beliefs are similar to the dynamics of PI beliefs. When the majority of previous guesses are $A$ and the private signal is $b$, participants' belief is greater than $50 \%$ (grey line) only after a depth of 2 but largely greater than $50 \%$ with a depth larger than 3 . The same phenomenon appears with a majority of previous $B$ guesses, albeit beliefs cross $50 \%$ only after a depth of 3 (instead of 2 in a sequence with a majority of $A$ ). This explains why participants rarely engage in cascade behavior with a depth of 1 but very frequently with a depth greater than 3 .

Similarly, the fact that the relative frequency of cascade break decreases with the depth of a cascade can also be explained by the way high informed participants update their beliefs. Figure 5 shows the dynamics of high informed participants' beliefs when the depth of the cascade increases and their signal contradicts the established pattern

\footnotetext{
${ }^{20}$ For depths strictly greater than 4 we only report the average of participants' beliefs since the number of observations falls from 117 with no previous guess to 15 with depth 5 . In this way, we consider 54 observations for depths strictly greater than 4 .
} 


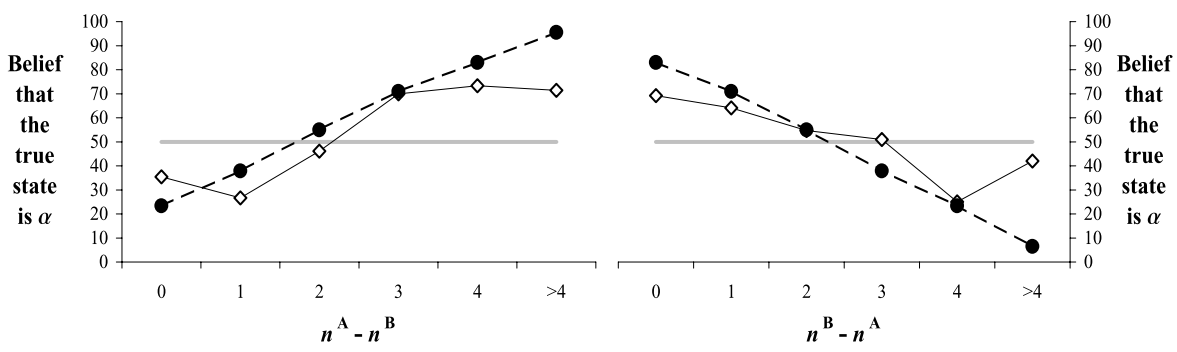

Fig. 5 Dynamics of beliefs with a strong contradictory signal (high line)

of guesses. Like in the low line, the dynamics of the high informed participants' beliefs are close to the dynamics of PI beliefs. In both cases (majority of previous $A$ guesses and majority of previous $B$ guesses), participants believe more in the state favored by the majority of previous guesses than in their own contradictory signal after a depth of 3 . This explains why participants rarely break a cascade of depth 3 or more even with a strong contradictory signal. ${ }^{21}$

\subsection{A statistical error-rate analysis}

A natural candidate to explain low informed participants' deviations from equilibrium are errors. If the likelihood of an error is inversely related to its cost then most deviations occur in situations where equilibrium behavior predicts a guess inconsistent with the one based only on the private signal. Indeed, in such situations, a deviation following a confirmatory signal is more costly than a deviation following a contradictory signal. Errors constitute a plausible rationalization to the overweightphenomenon, namely that low informed participants do not systematically engage in cascade behavior after having observed a few identical guesses. To rationalize the fact that low informed participants engage in cascade behavior after having observed many identical guesses, a further step in the reasoning process of the participants is necessary: Participants know that others make guess errors which are monotone in payoffs, they know that other participants know it, and so on. Generalizing the Bayesian Nash equilibrium by allowing for noisy optimizing behavior while maintaining the internal consistency of rational expectations defines McKelvey and Palfrey (1995) quantal response equilibrium (QRE) (in our context, the monotone agent quantal response equilibrium; see McKelvey and Palfrey 1998). Since the seminal work of AH, several experimental papers have suggested QRE as an alternative theory of behavior to Bayesian rationality, and the literature has focused on the logit specification (LQRE) because players' behavior is determined by a single parameter, the sensitivity to payoff differences, with a natural "rationality" interpretation. ${ }^{22}$ In

\footnotetext{
${ }^{21}$ Note that in both lines participants' guesses are highly consistent with their beliefs (about $97 \%$ of the time), i.e., participants maximize their expected payoff given their beliefs.

${ }^{22}$ Haile et al. (2008) show that evaluating the predictive success of QRE in a single game is uninformative without strong a priori restrictions on distributions of payoff perturbations. The logit specification imposes such restrictions and limits the number of observable phenomena in an information cascades game.
} 
LQRE, low informed players often guess according to their contradictory private signal after having observed a few identical guesses not only because of the small cost of the deviation but also because they discount the evidence conveyed by the observed guesses. Consequently, social learning never stops since low informed players do not fully discount the evidence conveyed by later guesses. Still, once a long sequence of identical guesses is observed, low informed players assign a large probability to the state favored by those guesses and they often engage in cascade behavior.

Though the dynamics of beliefs and guesses of low informed participants can be rationalized by LQRE, high informed participants do not "better respond" to rational expectations about the underlying distributions. Indeed, high informed participants endowed with a contradictory signal assign an extreme belief to the state favored by many identical guesses, which explains the low empirical probability of collapse, but such extreme beliefs are clearly at odds with the predictions of LQRE. ${ }^{23}$ In an attempt to rationalize low and high informed participants' behavior we conduct a depth-of-reasoning analysis, along the lines of Kübler and Weizsäcker (2004). We estimate an error-rate model that uses logistic response functions to determine guess probabilities and allows for different sensitivities to payoff differences on different levels of reasoning about others' behavior. ${ }^{24}$

Participants are assumed to make guess $A$ with probability

$$
\begin{aligned}
\operatorname{Pr} & \left(A \mid t_{i}, h^{i-1}, \lambda_{1}\right) \\
& =\frac{\exp \left(\lambda_{1}\left(15 \mu_{i}\left(t_{i}, h^{i-1}\right)-5\right)\right)}{\exp \left(\lambda_{1}\left(15 \mu_{i}\left(t_{i}, h^{i-1}\right)-5\right)\right)+\exp \left(\lambda_{1}\left(15\left(1-\mu_{i}\left(t_{i}, h^{i-1}\right)\right)-5\right)\right)},
\end{aligned}
$$

and guess $B$ with the complementary probability. The parameter $\lambda_{1}$ captures the sensitivity to payoff differences of the participants. Participants are assumed to attribute a sensitivity to payoff differences $\lambda_{2}$ to the guesses of the other participants. In addition, when participants consider the reasoning that other participants attribute to others' reasoning, they assign another sensitivity to payoff differences, $\lambda_{3}$. Additional higher-level sensitivities are assigned to longer chains of reasoning. Under the restriction that the data generating process is correctly specified by the Bayesian Nash equilibrium, the true value of the sensitivities should be infinity. Under the restriction that the data generating process is correctly specified by the LQRE, the true value of the sensitivities should be constant $\left(\lambda^{C}>0\right)$.

\footnotetext{
${ }^{23}$ This observation is true whatever the level of the sensitivity to payoff differences. If the sensitivity to payoff differences is large then high informed players infer much information from the first guesses (first one guess in an A herd and first two guesses in a B herd) but very little from later guesses. If the sensitivity to payoff differences is small then high informed players infer little information from any observed guess. In both cases, high informed participants always assign a higher belief to the state favored by their private signal unless an extremely long sequence of identical guesses is observed.

${ }^{24}$ The failure of LQRE to account for the documented regularities in social learning experiments has been often reported in the literature. Among others, Huck and Oechssler (2000) and Nöth and Weber (2003) point out that participants' reluctance to engage in cascade behavior is not solely due to their scepticism about others' capacity to learn in a Bayesian rational way. In a recent meta analysis of social learning experiments, Weizsäcker (2008) clearly demonstrates that QRE does not organize well the bulk of the experimental evidence.
} 
Table 7 shows the maximum likelihood estimation results. The top panel displays the results for LQRE and the bottom panel displays the results of the levelof-reasoning model. The left (right) panel shows the results on the data from the low (high) line. For each line, results are shown for the pooled data, and for experiment 1 and 2 separately. ${ }^{25}$ At the $5 \%$ significance level, sensitivities to payoff differences $\lambda_{1}$ and $\lambda_{2}$ are significantly different from 0 in all six subsets of the data. The sensitivity to payoff differences $\lambda_{3}$ is significantly different from 0 in all subsets of the data except from the high line in experiment 2 . The sensitivity to payoff differences $\lambda_{4}$ is not significantly different from 0 in all six subsets of the data. At the $5 \%$ significance level, sensitivities to payoff differences $\lambda_{1}$ and $\lambda_{2}$ are significantly different from the estimate of the sensitivity at the higher reasoning level, on both pooled data sets.

The results of our depth-of-reasoning analysis suggest that participants apply medium chains of reasoning. They learn from observing their predecessors' guesses and realize that other participants also learn from observing their respective predecessors. However, participants' reasoning gets rather imprecise on the highest level since participants think that others attribute to their respective predecessors twice the participants' error rate. Additionally, low informed participants attribute a significantly higher error rate to their predecessors whereas high informed participants attribute a significantly lower error rate to their predecessors as compared with their own. Thus, our estimation results confirm those of Kübler and Weizsäcker (2004) since low informed participants attribute a significantly lower "rationality" to their predecessors compared with their own. They also extend them since high informed participants attribute a significantly higher "rationality" to their low informed predecessors compared with their own which suggests that the perception of others' rationality is affected by the participant's information endowment. The reasoning process of low informed participants explains why they do not systematically engage in cascade behavior after having observed a few identical guesses but do so after having observed many identical guesses, and the reasoning process of high informed participants justifies the low empirical probability of collapse of long cascades.

\section{Discussion}

Experimental economists have established the anatomy of failure in social learning environments. After few identical guesses participants often follow their contradictory signal but they (almost) systematically engage in cascade behavior once many predecessors made the same guess. Several alternative theories of behavior to Bayesian rationality account for the prevalence of the overweight-phenomenon and its attenuation in long pure laboratory cascades. We present below the most prominent

\footnotetext{
${ }^{25}$ To construct hypotheses tests, we conduct a simulation study. We generate a set of simulated guesses, based on the maximum likelihood estimates and the sample size used in our experiment. We re-estimate the model and obtain a vector of maximum likelihood estimates of the simulated data. We repeat the process a large number of times. We obtain a distribution that resembles the sampling distribution of the maximum likelihood estimator. The standard errors are obtained by the sample standard deviation. Confidence intervals are obtained by deleting values of the appropriate number of estimates from the sorted array of maximum likelihood estimates.
} 
Table 7 Sensitivity to payoff differences estimates; $95 \%$ and $90 \%$ confidence intervals in brackets; negative log-likelihood (-LL). Parameter values in bold indicate sensitivities that are significantly different from 0 at conventional error levels. Parameter values with a subscript "a" ("b") indicate sensitivities that are significantly different from the corresponding common error rate, $\lambda^{C}$, at a $5 \%(10 \%)$ level. Parameter values with a subscript "c" indicate sensitivities that are significantly different from the estimate of the sensitivity at the lower reasoning level at the $5 \%$ level

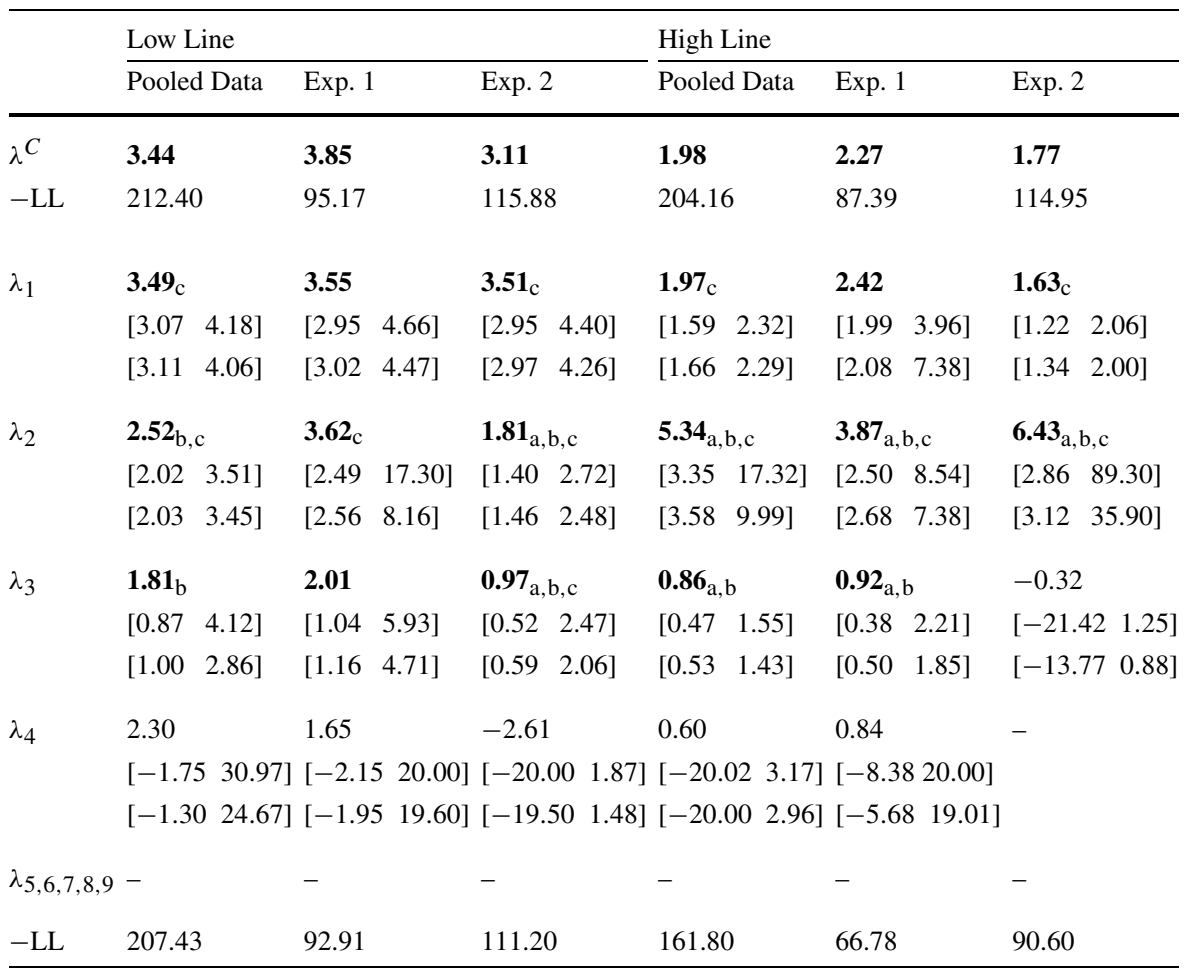

of these alternatives and we investigate which ones account for our new evidence. We end this section by discussing the actual allocative efficiency in both lines.

As already mentioned, LQRE has been suggested early in the experimental literature as an alternative theory of behavior to Bayesian rationality. LQRE accounts for the overweight-phenomenon and its attenuation in long pure laboratory cascades which means that its predictions are qualitatively in line with the behavior of our low informed participants. However, LQRE's predictions do not agree with the participants' behavior in the high line. There are basically two ways to modify LQRE in order to improve its predictive success in social learning experiments. In this paper, as initially suggested by Kübler and Weizsäcker (2004), we relax the rational expectations assumption and show that, compared with their own, low informed participants attribute a significantly lower rationality to their predecessors whereas high informed participants attribute a significantly higher rationality to their low informed predecessors. Not only do we confirm the earlier findings according to which the perception of others' rationality is biased but our estimation results suggest that this perception is affected by the participant's information endowment. A second possible extension 
of LQRE has been suggested by Goeree et al. (2007) and assumes that players update their beliefs in a non-Bayesian way which is commonly know. Players overweight their private information i.e. count their private signal as $k$ signals where $k \in(1, \infty)$ when forming their beliefs. Such an extension retains the rational expectations assumption but gives up the fact that players better respond. ${ }^{26}$ Our experimental results suggest that the overweight-parameter is not commonly known since high informed participants have different expectations than low informed participants about the rule used by low informed participants to update their beliefs. ${ }^{27}$

With the help of a meta data set of 13 experimental studies on BHW's specific model, Weizsäcker (2008) shows that participants are unsuccessful in learning from others in information cascade games since they underestimate the informational content of their predecessors' guesses. In situations where it is empirically optimal for participants to contradict their private signal, they make the appropriate guess in less than half of the cases. The behavior of low informed participants confirms this finding. We compare the proportion of correct guess among the actual guesses of the low informed participants with the proportion of correct guess among the guesses of Bayesian rational players who interpret a deviation from the equilibrium path as revealing the deviator's private signal. Overall, slightly more theoretical guesses are correct than actual guesses $(81.36 \%$ vs. $77.28 \%)$. The difference is entirely explained by the suboptimal play of low informed participants endowed with an incorrect private signal. If low informed participants would overweight their private information relative to the public information of their predecessors' guesses and successfully learn from others then they would do worst than Bayesian rational players early in the sequence but would do better over time as more and more information gets revealed. In fact, the accuracy of actual guesses does not improve over time compared to the accuracy of theoretical guesses. In the first four periods (respectively last four periods) of the decision making sequence, the proportion of correct theoretical guesses is $12 \%$ higher (respectively 14\% higher) than the proportion of correct guesses made by low informed participants endowed with an incorrect private signal. The proportion of correct theoretical guesses is almost identical to the proportion of correct guesses made by low informed participants endowed with a correct private signal at any point in the decision making sequence. On the contrary, slightly less theoretical guesses are correct than actual guesses made by high informed participants $(76.19 \%$ vs. $78.25 \%)$ and the improvement in guess accuracy only occurs in situations where high informed participants are endowed with an incorrect private signal. Overall, the proportion of correct theoretical guesses is $45 \%$ lower than the proportion of correct guesses made

\footnotetext{
${ }^{26}$ March and Ziegelmeyer (2009) generalize this extension by assuming that players either overweigh or underweigh (in Bayesian terms) their private information relative to the public information revealed by the decisions of others.

${ }^{27}$ Two recent theoretical papers derive predictions in numerous social learning environments assuming that players are boundedly rational. Guarino and Jehiel (2009) investigates the behavior of players who choose according to the Analogy Based Expectation Equilibrium using the payoff-relevant analogy partition (this implies that there are only two analogy classes, one for each state). Eyster and Rabin (2009) investigates the implications of naïve herding according to which players believe that each predecessor's guess reflects solely his private information. Both approaches predict that the beliefs of players become extreme when they observe long sequences of identical guesses and the low empirical probability of collapse of long herds. However, both approaches fail to explain the overweight-phenomenon.
} 
by high informed participants endowed with an incorrect private signal (it is $17 \%$ lower in the third period and $72 \%$ lower in the ninth period). The proportion of correct theoretical guesses is $11 \%$ higher than the proportion of correct guesses made by high informed participants endowed with a correct private signal (it is $7 \%$ higher in the third period and $12 \%$ higher in the ninth period). In contrast with low informed participants, high informed participants are successful in learning from others and they behave efficiently when engaging in cascade behavior.

\section{Concluding remarks}

We examine the occurrence and fragility of information cascades in two laboratory experiments. In each decision making round, a random choice is made between two (almost) equally likely states but the state is not revealed to the participants until the end of the round. Low informed participants sequentially guess which state has been chosen, with each participant observing all previous guesses and a signal correct with probability $2 / 3$. In a matched pairs design, high informed participants observe the guesses of the low informed participants and a signal correct with probability 4/5. In the second experiment, participants' beliefs about the chosen state are elicited.

The fragility of laboratory cascades rapidly vanishes as the number of identical guesses increases. Said differently, the behavior of high informed participants deviates from equilibrium behavior in situations where Bayesian rationality predicts a guess consistent with the one based only on the private signal. This result complements the main finding in earlier experimental cascade games, the overweightphenomenon, according to which participants' behavior deviates from equilibrium behavior essentially in situations where Bayesian rationality predicts a guess inconsistent with the one based only on the private signal. Additionally, earlier experimental studies have established that participants' tendency to engage in cascade behavior increases with the number of identical guesses i.e. participants endowed with weak contradictory signals guess more in accordance with Bayesian rationality when the evidence conveyed by previous guesses is strong. Due to this behavioral pattern, some authors have interpreted their experimental data as a clear support for the equilibrium prediction (among others, Anderson and Holt 1997 and Hung and Plott 2001). We show that participants endowed with strong contradictory signals guess less in accordance with the equilibrium prediction when the evidence conveyed by previous guesses is strong. This evidence is corroborated by the analysis of participants' elicited beliefs which show that, unlike in equilibrium, the more cascade guesses participants observe the more they believe in the state favored by those guesses.

Compared to Bayesian rational players, low informed participants guess suboptimally when endowed with an incorrect private signal since they underestimate the informational content of their predecessors' guesses. In contrast, high informed participants are successful in learning from others and the remarkable stability of laboratory cascades reflects their efficient behavior. Further experimental studies should investigate the efficiency of participants' behavior in social learning environments with long decision making sequences and heterogeneous signal accuracies. 
Acknowledgements The authors have benefited from comments and suggestions from Mohammed Abdellaoui, Nick Feltovich, John Hey, Dorothea Kübler, Bradley Ruffle, Georg Weizsäcker, Marc Willinger, participants at F.U.R. X in Torino, 5ièmes Journées d'Economie Expérimentale in Strasbourg, and seminars in Ben Gurion University, University College London, Hebrew University of Jerusalem, University of Edinburgh, and Rice University. We are indebted for the financial support provided by the Department of Economics at the University of Strasbourg. The editor, Jordi Brandts, and two anonymous referees provided remarks that improved the manuscript.

Open Access This article is distributed under the terms of the Creative Commons Attribution Noncommercial License which permits any noncommercial use, distribution, and reproduction in any medium, provided the original author(s) and source are credited.

\section{References}

Acemoglu, D., Dahleh, M., Lobel, I., \& Ozdaglar, A. (2008). Bayesian learning in social networks (Working Paper 2780). mIT LIDS.

Alevy, J., Haigh, M., \& List, J. (2007). Information cascades: Evidence from a field experiment with financial market professionals. Journal of Finance, 62, 151-180.

Anderson, L. (2001). Payoff effects in information cascade experiments. Economic Inquiry, 39, 609-615.

Anderson, L., \& Holt, C. (1997). Information cascades in the laboratory. American Economic Review, 87, 847-862.

Banerjee, A. (1992). A simple model of herd behavior. Quarterly Journal of Economics, 107, 797-818.

Bikhchandani, S., Hirshleifer, D., \& Welch, I. (1992). A theory of fads, fashion, custom, and cultural change as informational cascades. Journal of Political Economy, 100, 992-1026.

Blanco, M., Engelmann, D., Koch, A. K., \& Normann, H. T. (2008). Belief elicitation in experiments: Is there a hedging problem? (iZA DP No. 3517).

Celen, B., \& Kariv, S. (2004). Distinguishing informational cascades from herd behavior in the laboratory. American Economic Review, 94, 484-497.

Chamley, C. P. (2004). Rational herds: Economic models of social learning. Cambridge: Cambridge University Press.

Cipriani, M., \& Guarino, A. (2005). Herd behavior in a laboratory financial market. American Economic Review, 95, 1427-1443.

Drehmann, M., Oechssler, J., \& Roider, A. (2005). Herding and contrarian behavior in financial markets: An Internet experiment. American Economic Review, 95, 1403-1426.

Eyster, E., \& Rabin, M. (2009). Rational and naive herding (cEPR Discussion Paper 7351). London: Centre for Economic Policy Research.

Goeree, J., Palfrey, T., Rogers, B., \& McKelvey, R. (2007). Self-correcting information cascades. Review of Economic Studies, 74, 733-762.

Guarino, A., \& Jehiel, P. (2009). Social learning with coarse inference (Mimeo).

Haile, P., Hortacsu, A., \& Kosenok, G. (2008). On the empirical content of quantal response equilibrium. American Economic Review, 98, 180-200.

Huck, S., \& Oechssler, J. (2000). Informational cascades in the laboratory: Do they occur for the right reasons? Journal of Economic Psychology, 21, 661-671.

Hung, A., \& Plott, C. (2001). Information cascades: Replication and an extension to majority rule and conformity rewarding institutions. American Economic Review, 91, 1508-1520.

Koessler, F., \& Ziegelmeyer, A. (2000). Tie-breaking rules and informational cascades: a note (Working Paper BETA 2000-09). Université Louis Pasteur, Strasbourg.

Kraemer, C., Nöth, M., \& Weber, M. (2006). Information aggregation with costly information and random ordering: Experimental evidence. Journal of Economic Behavior \& Organization, 59, 423-432.

Kübler, D., \& Weizsäcker, G. (2004). Limited depth of reasoning and failure of cascade formation in the laboratory. Review of Economic Studies, 71, 425-441.

Kübler, D., \& Weizsäcker, G. (2005). Are longer cascades more stable? Journal of the European Economic Association, 3, 330-339. (Papers and Proceedings).

March, C., \& Ziegelmeyer, A. (2009). Behavioral social learning (Mimeo).

McKelvey, R., \& Palfrey, T. (1995). Quantal response equilibria for normal form games. Games and Economic Behavior, 10, 6-38. 
McKelvey, R., \& Palfrey, T. (1998). Quantal response equilibria for extensive form games. Experimental Economics, 1, 9-41.

Murphy, A., \& Winkler, R. (1970). Scoring rules in probability assessment and evaluation. Acta Psychologica, 34, 273-286.

Nöth, M., \& Weber, M. (2003). Information aggregation with random ordering: Cascades and overconfidence. Economic Journal, 113, 166-189.

Nyarko, Y., \& Schotter, A. (2002). An experimental study of belief learning using elicited beliefs. Econometrica, 70, 971-1005.

Offerman, T., Sonnemans, J., van de Kuilen, G., \& Wakker, P. (2009). A truth-serum for non-bayesians: Correcting proper scoring rules for risk attitudes. Review of Economic Studies, 76, 1461-1489.

Siegel, S., \& Castellan, N. J. (1988). Nonparametric statistics for the behavioral sciences. New York: McGraw-Hill.

Smith, L., \& Sorensen, P. (2000). Pathological outcomes of observational learning. Econometrica, 68, 371-398.

Weizsäcker, G. (2008, forthcoming). Do we follow others when we should? A simple test of rational expectations. American Economic Review.

Ziegelmeyer, A. (2001). Informational externalities and sequential interactions: experimental evidence on social learning. Unpublished Ph.D. thesis. Louis Pasteur University, Strasbourg. 